\title{
Medication Adherence and Effective Management of Hypertension
}

\author{
Seung-Won $\mathrm{Oh}^{*}$ \\ Department of Family Medicine, Seoul National University Hospital Healthcare System Gangnam Center, Seoul, Korea
}

\section{See original paper on 150}

The 2018 Korea National Health and Nutrition Examination Survey (KNHANES) revealed that the prevalence of hypertension was $28.3 \%$ among adults aged 30 years or older, and the awareness, treatment, and control rates of hypertension among hypertensive patients were $65 \%, 61.1 \%$, and $45.4 \%$, respectively. Even among patients treated with antihypertensive medications, $26.5 \%$ could not reduce their blood pressure to a level of $<140 / 90 \mathrm{~mm} \mathrm{Hg.}{ }^{1)}$ Although the indicators of the control of hypertension have been steadily improving in recent years, they are not yet satisfactory. Poor adherence to prescribed medications is thought to be the most important cause of insufficient blood pressure control.

In the present study, Khadoura et al. ${ }^{2)}$ investigated the prevalence of antihypertensive medication non-adherence and factors associated with it among 538 Iranian hypertensive patients attending primary clinics. In this cross-sectional survey, the prevalence of non-adherence was $65.8 \%$. Old age, high education level, low frequency and number of medication, and fewer comorbidities were associated with good adherence. Additionally, patients with high self-efficacy and social support had high adherence to antihypertensive medication.

Drug compliance is also an important issue in the treatment of hypertensive patients in Korea. To increase the effectiveness of antihypertensive treatment, an approach to figure out factors that reduce drug compliance is needed. The appropriate adherence rate was only $54.7 \%$ in the study using pharmacy claims data and qualification data of the insured of the national health insurance form in 2004. Upon multiple logistic regression analysis, the probability of appropriate medication adherence decreased in females, with age, when pa- tients have a disability, prescription days per visit decreased, and the number of prescribing physicians increased. ${ }^{3)}$ A study using the $2008 \mathrm{KNHANES}$ data reported that cases with older age, a spouse, medicare insurance, a higher number of cormorbidities, and no current smoking status showed significantly high medication adherence. ${ }^{4)}$ Choi et al. ${ }^{5}$ investigated factors affecting adherence to antihypertensive medication in 1,523 Korean hypertensive patients who visited family physicians of primary care clinics and hospitals. They reported that age $\geq 65$ years, concomitant medication for diabetes, a family history of hypertension and/or cardiovascular disease, being on $\geq 2$ classes of antihypertensive medications, high frequency of exercise, and treatment in a metropolitan-located hospital were associated with good adherence, while a high salt intake was associated with poor adherence.

The differences in the characteristics or environment of the subjects between each study would be a reason for the large variety of factors that affect adherence. For example, studies between home blood pressure monitoring and drug compliance didn't show consistent results. ${ }^{6,7)}$ Eventually, more diverse studies will be needed on factors that affect drug compliance, and access based on patient characteristics might be required for effective management. In addition, to increase the control rates, which are still low in Korea, attention will also need to be paid to other factors affecting uncontrolled hypertension. $^{8)}$

\section{CONFLICT OF INTEREST}

No potential conflict of interest relevant to this article was reported. 


\section{ORCID}

Seung-Won Oh: https://orcid.org/0000-0003-3800-0754

\section{REFERENCES}

1. Korean Society of Hypertension (KSH), Hypertension Epidemiology Research Working Group. Korea hypertension fact sheet 2020. Clin Hypertens 2020 (in press).

2. Khadoura K, Shakibazadeh E, Mansournia M, Aljeesh Y, Fotouhi A. Determining the prevalence of and the factors associated with antihypertensive medication non-adherence in the Gaza Strip. Korean J Fam Med 2021;42:150-8.

3. Park JH, Shin Y, Lee SY, Lee SI. Antihypertensive drug medication adherence and its affecting factors in South Korea. Int J Cardiol 2008;128: 392-8.

4. Cho E, Lee CY, Kim I, Lee T, Kim GS, Lee H, et al. Factors influencing medication adherence in patients with hypertension: based on the 2008 Korean National Health and Nutrition Examination Survey. J Korean Acad Community Health Nurs 2013;24:419-26.

5. Choi HY, Oh IJ, Lee JA, Lim J, Kim YS, Jeon TH, et al. Factors affecting adherence to antihypertensive medication. Korean J Fam Med 2018; 39:325-32.

6. Muhammad J, Jamial MM, Ishak A. Home blood pressure monitoring has similar effects on office blood pressure and medication compliance as usual care. Korean J Fam Med 2019;40:335-43.

7. Ogedegbe G, Schoenthaler A. A systematic review of the effects of home blood pressure monitoring on medication adherence. J Clin Hypertens (Greenwich) 2006;8:174-80.

8. Kwon J, Lim CY, Kim M. Uncontrolled blood pressure in hypertensive patients with high medication adherence: a Korean nationwide population-based study. Korean J Fam Med 2020;41:28-37. 